\title{
Clinical Progress Note: Vascular Access Appropriateness Guidance for Pediatric Hospitalists
}

\author{
Katie A Meier, MD ${ }^{1,2 *}$, Rajendu Srivastava, MD, $\mathrm{MPH}^{3,4}$
}

\begin{abstract}
${ }^{1}$ Department of Pediatrics, University of Cincinnati College of Medicine, Cincinnati, Ohio; ${ }^{2}$ Division of Hospital Medicine, Cincinnati Children's Hospital Medical Center, Cincinnati, Ohio; ${ }^{3}$ Division of Inpatient Medicine, Department of Pediatrics, University of Utah and Intermountain Primary Children's Hospital, Salt Lake City, Utah; ${ }^{4}$ ntermountain Healthcare Delivery Institute, Murray, Utah.
\end{abstract}

ospitalized pediatric patients often require vascular access for necessary therapies, such as antibiotics. However, vascular access devices (VADs) are also associated with harm, ranging from insertion complications to life-threatening bloodstream infections or thrombosis. ${ }^{1}$ Pediatric hospitalists often guide VAD placement. There is a paucity of evidence to guide VAD selection based on the relative benefits and risks. ${ }^{2}$ The Michigan Appropriateness Guide for Intravenous Catheters in Pediatrics (miniMAGIC) ${ }^{2}$ offers the first set of standards. Like its adult predecessor guideline (MAGIC) published in 2015, it provides guidance on appropriate VAD selection based on current evidence and expertise from a multidisciplinary panel. ${ }^{2}$ The guideline informs device selection, device characteristics, and insertion technique for the pediatric population (term neonates to adolescents) and across a wide range of clinical indications. ${ }^{2}$ This review highlights key recommendations for pediatric hospitalists to help their decision-making.

\section{METHODS USED IN PREPARING THE GUIDELINE}

miniMAGIC was developed using the RAND/UCLA Appropriateness Method, a method proven to reduce inappropriate (ie, overused or underused) healthcare interventions. ${ }^{3}$ It combines rigorous evidence review with multidisciplinary expert opinion on real-world clinical scenarios to provide recommendations about an intervention's appropriateness. ${ }^{3}$ This is particularly useful for clinical scenarios that lack high-quality evidence to guide decision-making. The RAND/ UCLA method deems an intervention appropriate if the benefits outweigh the risks by a wide enough margin that proceeding is worthwhile, and it does not take cost into account. ${ }^{2}$ The method design consists of five phases: (1) defining the scope and key terms, (2) reviewing and synthesizing the literature, (3) selecting an expert panel, (4) developing case scenarios, and (5) conducting two rounds of appropriateness ratings by the expert panel for each clinical scenario. ${ }^{3}$ The guideline's scope included term neonates (aged 0-30 days),

*Corresponding Author: Katie A Meier, MD; Email: katie.meier@cchmc.org; Telephone: 513-803-9177; Twitter: @KMeierMD.

Published online first April 20, 2021

Find additional supporting information in the online version of this article.

Received: July 31, 2020; Revised: January 17, 2021; Accepted: January 20, 2021

(C) 2021 Society of Hospital Medicine DOI 10.12788/jhm.3595 infants (aged 31 days-1 year), children (aged 1-12 years), and adolescents (aged 12-18 years). Infants receiving care in the neonatal intensive care unit or special care nursery were excluded. Other specialized populations addressed based on setting or diagnosis were general hospitalized patients and patients with congenital cardiac disease, critical illness, oncologic and hematologic conditions, and long-term VAD-dependent conditions. ${ }^{3}$

A total of 133 studies or clinical practice guidelines (CPGs) met the eligibility criteria for the systematic review. ${ }^{4}$ Although the systematic review was conducted per the RAND/UCLA method using two independent reviewers who evaluated the methodologic quality, transparency, and relevancy of each article, there was no formal assessment of evidence quality. The recommendations were based primarily on observational studies and CPGs because there were few randomized controlled trials or systematic reviews on VAD selection for pediatric patients in the literature. Pediatric evidence was limited for certain scenarios or populations (eg, term neonates, midline catheters, difficult venous access, long-term VAD), so adult and/or neonatal evidence was included when applicable.

The panel included 14 pediatric clinical experts from cardiology, vascular access, critical care, hematology/oncology, emergency medicine, general surgery, hospital medicine, anesthesia, interventional radiology, pharmacology, and infectious diseases. The panel also included nonvoting panel members such as the panel facilitators, a methodologist, and a patient representative.

\section{RESULTS OF THE CLINICAL REVIEW}

We review four common clinical scenarios encountered by pediatric hospitalists and summarize key recommendations (Table).

\section{Peripherally Inserted Central Catheter}

Patients may require peripherally inserted central catheters (PICCs) to facilitate a longer duration of intravenous (IV) therapy, such as delivery of antibiotics, or frequent blood draws. The need for prolonged vascular access is decreasing, as studies show many infections in children previously treated with prolonged IV antibiotics can be safely and equally effectively managed with early transition to oral therapy. ${ }^{5-8}$ These studies highlight the higher rate of complications and risks associated with PICCs, including thrombosis, infection, and mechanical issues, as well as the added healthcare utilization needed to evaluate 
TABLE. Key Recommendations for Pediatric Hospital Medicine ${ }^{2,11}$

PICC placement is inappropriate for any peripheral therapy lasting $<8 \mathrm{~d}$, regardless of patient age.

PICC placement is appropriate for

- Nonperipheral therapy of any length;

- Peripheral therapy lasting $>14 \mathrm{~d}$ in infants, children, adolescents and $>7 \mathrm{~d}$ in neonates;

- Frequent blood draws for $>14 \mathrm{~d}$ in children/adolescents and $>7 \mathrm{~d}$ in neonates/infants or if PICC is $\geq 3 \mathrm{~F}$ regardless of age.

Consider in patients with difficult access no matter the length of anticipated need.

Midline catheter placement is another option for peripheral therapy or frequent blood draws, particularly for neonates and adolescents, but more research is needed.

In the case of difficult access,

- Escalate early to an advanced inserter after no more than two peripheral IV attempts;

- Consider PICC placement no matter the length of anticipated need;

- Use ultrasound to aid peripheral IV or PICC placement.

For long-term vascular access decisions,

- Partner with patient/family in VAD selection;

- Do not use hospitalization frequency as a defining criterion;

Take into account infusate characteristics and therapy length/intermittency when selecting a VAD.

Abbreviations: IV, intravenous; PICC, peripherally inserted central catheter

$V A D$, vascular access device.

and manage the complications. PICC-associated complication rates also increase with duration. ${ }^{4}$

However, there are some clinical scenarios that still warrant prolonged therapy and/or access; PICC recommendations are summarized in the Appendix Figure. The miniMAGIC panel deemed PICC lines appropriate for any nonperipheral therapy of any length. For peripherally compatible therapy, the panel rated PICC placement as inappropriate for therapy lasting less than 8 days, regardless of patient age. PICC placement in infants, children, and adolescents was rated appropriate for therapy with a duration exceeding 14 days, but the panel was uncertain about therapy expected to last between 8 and 14 days. Recognizing the additional challenges with maintaining peripheral IV catheter access in neonates, PICCs were deemed appropriate for neonates needing peripheral therapy lasting longer than 7 days.

The panel rated PICC placement appropriate for frequent blood draws (defined as more than one time per day) for more than 7 days in neonates or infants and more than 14 days in children and adolescents. But regardless of patient age, the PICC caliber must be at least 3F.

The miniMAGIC panel found that a single lumen is appropriate in most cases, highlighting that multilumen catheters increase the risk for infection, occlusion, and venous thrombosis. ${ }^{4}$ Multilumen catheters were rated as inappropriate in the case of reserving a lumen for blood products and blood sampling. When reserving a lumen for lipids and parenteral nutrition (PN), the panel was uncertain given the lack of evidence regarding the risks/benefits of the complications associated with the infusions themselves versus those of the device. Regardless, collaboration with a pharmacist and vascular access specialist is recommended to aid in choosing the most appropriate device characteristics.

\section{Midline Catheters}

Midline catheters are inserted in a peripheral vein, but the catheter tip terminates in the proximal extremity. Compared with peripheral IV catheters, midline catheters last longer and have lower rates of phlebitis. In addition, midline catheter placement does not require sedation or fluoroscopy and has lower rates of infection compared with PICC lines. ${ }^{9} \mathrm{Al}-$ though there is good evidence in adults, and multiple panelists reported success in using midline catheters in various age groups, the evidence for their safe and efficacious use in pediatrics is limited, particularly for infants. Midline catheters were rated as appropriate for peripheral therapy lasting less than 8 days in neonates and less than 15 days in children and adolescents. Use in infants was deemed uncertain based on lack of published evidence. Midline catheters were also rated as appropriate for frequent blood draws of less than 8 days in neonates and less than 15 days in adolescents, but uncertain for children and infants.

\section{Difficult Access and Insertion Procedure}

The panel rated three or more attempts for peripheral IV catheter insertion by a single clinician as inappropriate and recommended early escalation to a more experienced inserter after 0 to 2 attempts by a single provider. The goal is to preserve insertion sites and reduce patient discomfort. If a patient loses access when only 1 day of therapy remains, the provider should transition to oral or intramuscular therapy when appropriate, particularly if there are no advanced insertion staff available or after two or more attempts at re-insertion are unsuccessful. There is high-quality evidence that supports vessel visualization (primarily ultrasound) with peripheral IV catheter and PICC placement. ${ }^{2}$ In the case of two or more unsuccessful attempts at peripheral IV catheter placement by an advanced inserter using technology assistance (ultrasound), PICC placement is considered appropriate by the panel to avoid delays in treatment and limit patient discomfort associated with repeat attempts.

\section{Long-term Vascular Access}

Children with medical complexity or chronic illness may require long-term ( $>2$ months) or very-long-term ( $>1$ year) vascular access. Common themes for VAD selection in this heterogeneous population include a focus on vessel preservation and complication prevention. ${ }^{2}$ The panel strongly recommended that clinicians partner with the patient and caregivers in the decision-making process. Shared decision-making is necessary to meet both the short-term and evolving needs of the of the patient and family. The panel also believed the frequency of hospitalization should not be used as a criterion for VAD selection since acute hospitalization is an unreliable proxy for disease severity in pediatric chronic disease conditions. ${ }^{2}$ Rather, the infusate characteristics and length/intermittency of therapy should be primary influencers of VAD selection. In general, the panel rated cuffed tunneled central VADs (CVADs) as appropriate for all age groups for long-term PN, long-term continuous infusions, 
and long-term intermittent therapies. For continuous non-PN infusions, appropriate ratings were given to PICCs for infants and children and total implanted venous devices (TIVDs) in children and adolescents. For intermittent (but at least daily) access, TIVDs and PICC lines were both rated as appropriate for children and adolescents but uncertain for neonates and infants. Peripheral devices were deemed inappropriate for all long-term complex therapies. For children and adolescents needing intermittent, regular peripheral treatments (eg, steroids or antibiotics), peripheral IVs and TIVDs were rated appropriate for short duration ( $<7$ days) therapies. PICCs and midlines for this indication were uncertain because of the lack of evidence. For medium-duration intermittent therapies (8-14 days), PICCs, tunneled cuffed CVADs, and TIVDs were rated as appropriate. A recently released mobile application can help guide the clinician through many varied clinical scenarios and indications. ${ }^{10}$

\section{LIMITATIONS AND GAPS}

The guideline recommendations were more often reliant on clinical practice guidelines and expert panel opinion given the lack of high-quality pediatric evidence for most scenarios. The panel members were from the United States and Australia, so the recommendations may not be generalizable to care systems in other countries. Although the panel included experts from many specialties that care for patient populations needing VADs, not all subspecialty populations were considered, particularly those with long-term vascular access-dependent conditions who may be commonly hospitalized. Scenarios with disagreement or uncertainty highlight gaps in need of future study (eg, midline catheter use and device selection for blood draws).

\section{CONCLUSIONS AND APPLICATION}

miniMAGIC is the first appropriateness guideline to help standardize the safe use of VADs in children. Although some gaps remain, the authors intend it to be a living document that will need revisions as new evidence is published. A mobile health application facilitates use of the recommendations, providing quick, point-of-care decision support based on clinical features. ${ }^{10}$ Pediatric hospitalists should collaborate with their institutions to examine their current VAD use in hospitalized children and identify opportunities for practice change and standardization. Use of these recommendations may help hospitalists improve the care of hospitalized children by decreasing unnecessary PICC placement and better partner with patients and caregivers to limit discomfort surrounding VAD placement.

Disclosures: The authors have nothing to disclose.

\section{References}

1. Ullman AJ, Marsh N, Mihala G, Cooke M, Rickard CM. Complications of central venous access devices: a systematic review. Pediatrics. 2015;136(5):e1331-e1344. https://doi.org/10.1542/peds.2015-1507
2. Ullman AJ, Bernstein SJ, Brown E, et al. The Michigan appropriateness guide for intravenous catheters in pediatrics: miniMAGIC. Pediatrics. 2020;145(Suppl 3):S269-S284. https://doi.org/10.1542/peds.2019-3474I

3. Ullman AJ, Chopra V, Brown E, et al. Developing appropriateness criteria for pediatric vascular access. Pediatrics. 2020;145(Suppl 3):S233-S242. https:// doi.org/10.1542/peds.2019-3474G

4. Paterson RS, Chopra V, Brown E, et al. Selection and insertion of vascular access devices in pediatrics: a systematic review. Pediatrics. 2020;145(Suppl 3):S243-S268. https://doi.org/10.1542/peds.2019-3474H

5. Keren R, Shah SS, Srivastava R, et al. Comparative effectiveness of intravenous vs oral antibiotics for postdischarge treatment of acute osteomeyelitis in children. JAMA Pediatr. 2015;169(2):120-128. https://doi.org/10.1001/ jamapediatrics.2014.2822

6. Shah SS, Srivastava R, Wu S, et al. Intravenous versus oral antibiotics for postdischarge treatment of complicated pneumonia. Pediatrics. 2016;138(6):e20161692. https://doi.org/10.1542/peds.2016-1692

7. Rangel SJ, Anderson BR, Srivastava R, et al. Intravenous versus oral antibiotics for the prevention of treatment failure in children with complicated appendicitis: has the abandonment of peripherally inserted catheters been justified? Ann Surg. 2017;266(2):361-368. https://doi.org/10.1097/ SLA.0000000000001923

8. Desai S, Aronson PL, Shabanova $\mathrm{V}$, et al. Parenteral antibiotic therapy duration in young infants with bacteremic urinary tract infections. Pediatrics. 2019;144(3):e20183844. https://doi.org/10.1542/peds.2018-3844

9. Anderson J, Greenwell A, Louderback J, Polivka BJ, Herron Behr J. Comparison of outcomes of extended dwell/midline peripheral intravenous catheters and peripherally inserted central catheters in children. J Assoc Vasc Access. 2016;21(3):158-164. https://doi.org/10.1016/j.java.2016.03.007

10. miniMAGIC: the Michigan Appropriateness Guide for Intravenous Catheters in Pediatrics app. Version 1.0.0. Alliance for Vascular Access Teaching and Research.

11. Shaughnessy EE, Morton K, Shah SS. Vascular access in hospitalized children. Pediatrics. 2020;145(Suppl 3):S298-S299. https://doi.org/10.1542/ peds.2019-3474P 